\title{
Effect of charred Radix et Rhizoma Rhei in a laser-induced choroidal neovascularization murine model
}

\author{
DONGMEI HAN, YUAN YAO, YONG SUN, YUANYUAN GONG and XINGWEI WU \\ Department of Ophthalmology, Shanghai First People's Hospital, School of Medicine, \\ Shanghai Jiao Tong University, Shanghai 200080, P.R. China
}

Received February 14, 2014; Accepted November 4, 2014

DOI: $10.3892 / \mathrm{mmr} .2014 .3046$

\begin{abstract}
A pharmaceutical composition (patent no. WO2012079419) exhibited favorable outcomes in a clinical trial of wet age-related macular degeneration. The aims of the present study were to explore the effects of one composition component, charred Radix et Rhizoma Rhei (CRRR), in a laser-induced choroidal neovascularization (CNV) murine model. A total of 30 eight-week-old C57BL/6 mice were subjected to diode laser treatment, and CNV was induced by rupturing the Bruch's membrane. The mice were then randomly divided into two groups: the CRRR-treated group that was administered CRRR water extract (concentration, $0.6 \mathrm{~g} / 100 \mathrm{ml}$; dose, $1 \mathrm{ml} / 0.1 \mathrm{~kg}$ twice a day for 21 days); and the control group that was treated with saline (dose, $1 \mathrm{ml} / 0.1 \mathrm{~kg}$ twice a day for 21 days). The retinal tissue was subjected to quantitative polymerase chain reaction (qPCR) and western blot analysis to determine the expression levels of interleukin-10 (IL-10) and vascular epithelial growth factor (VEGF) at day seven following laser treatment. At weeks 2 and 3 after laser treatment, fundus fluorescein angiography was performed and graded to assess the severity of lesion leakage. Retinal flat mounts were prepared for three-dimensional confocal microscopy at day 22 after laser treatment. At days 14 and 21 after laser treatment, no statistically significant differences were observed between the clinically relevant lesions of the CRRR-treated and control mice. CNV volumes were not found to be significantly different between the CRRR-treated and control mice. The expression levels of IL-10 were significantly increased in the CRRR-treated mice $(\mathrm{P}<0.05)$. However, no statistically significant differences were observed between the VEGF expression levels of the CRRR-treated and control
\end{abstract}

Correspondence to: $\mathrm{Dr}$ Xingwei $\mathrm{Wu}$, Department of Ophthalmology, Shanghai First People's Hospital, School of Medicine, Shanghai Jiao Tong University, 85 Wujin Road, Shanghai 200080, P.R. China

E-mail: wuxingwei2014@126.com

Key words: charred Radix et Rhizoma Rhei, choroidal neovascularization, traditional Chinese medicine, age-associated macular degeneration, interleukin-10 mice. In conclusion, CRRR did not appear to significantly inhibit CNV in this murine model. The function of CRRR in the pharmaceutical composition may be due to the effects of IL-10 and a synergistic effect with other components of the composition. However, further investigation is required.

\section{Introduction}

Age-associated macular degeneration (AMD) is the leading cause of legal blindness among individuals aged $>60$ years in developed countries (1). AMD is divided into two subtypes: Wet and dry. The development of choroidal neovascularization (CNV) is a key feature of wet AMD, which induces bleeding and scar formation in the macula and severely reduces the vision of patients (1). To date, no cure exists for AMD. Since 2003, numerous agents targeting vascular endothelial growth factors (VEGFs) have been approved by the US Food and Drug Administration for the treatment of CNV in AMD (2). Targeting VEGFs is an effective treatment method; however, there are concerns regarding repeated injections, CNV recurrence and the safety of long term application. Although VEGF plays an important role in the development of CNV, other mechanisms are also involved in this disorder (3). Alternative agents for the treatment of CNV are currently investigated, in order to improve their efficacy and safety and ensure that they are non-invasive. In 2012, Jin et al(4) devised a novel traditional Chinese medicine (TCM) composition for wet AMD (patent no. WO2012079419). This pharmaceutical composition was shown to enhance and stabilize the vision of patients with AMD, promote absorption of hemorrhage and reduce $\mathrm{CNV}$ fluorescein leakage and size (5). The pharmaceutical composition consisted of eight TCM components, including Astragalus membranaceus Bunge, Angelica sinensis, Poria cocos Wolf, Fritillaria thunbergii, Panax pseudoginseng, charred Radix et Rhizoma Rhei (CRRR), pollen Typhae and Curcuma aromatica Salisb. In addition, Jin et al (4) (patent no., WO2012079419) tested another composition for the treatment of CNV in AMD for four years; however, the clinical outcomes of the study were unsatisfactory. The previously used composition included Astragalus membranaceus, Angelica sinensis, Panax pseudoginseng, pollen Typhae, Citrus reticulata, Fritillaria thunbergii, Curcuma aromatic Salisb, Fructus and Poria cocos Wolf. The significant alteration between the previous and the present (WO2012079419) 
composition was the replacement of Citrus reticulata with CRRR. Thus, CRRR may play a vital role in the treatment of wet AMD. To the best of our knowledge, currently no publication exists regarding the mechanism of CRRR in this CNV treatment method. The aim of the present study was to explore the effect of CRRR treatment in a CVD murine model.

\section{Materials and methods}

Animals. All the animal experiments conformed to the Association for Research in Vision and Ophthalmology guide (www.arvo.org/About_ARVO/Policies/Statement_for_the_ Use_of_Animals_in_Ophthalmic_and_Visual_Research/) for the Use of Animals in Ophthalmic and Vision Research and were approved by the Institutional Animal Care and Use Committee of Shanghai Jiao Tong University, Shanghai, China (permit no. 2014KY110). A total of 30 male C57BL/6 mice (eight-weeks-old; Laboratory Animal Center of Shanghai Jiao Tong University) were used in the present study. The mice were fed normal mouse chow and supplied with water ad libitum. Prior to laser treatment and during subsequent retinal imaging, the mice were anesthetized by intraperitoneal (i.p.) injection of sodium pentobarbital (40 mg/kg; Sigma-Aldrich, St. Louis, MO, USA) and the pupils were dilated using topical $1 \%$ tropicamide (Beijing Double-Crane Pharmaceutical Co., Ltd., Beijing, China).

Laser-induced CNV in mice. Laser treatment was performed on the retinas of the mice. A coverslip was mounted onto the mouse cornea in order to view the retina and perform laser burns. Six laser burns were performed between the retinal vessels around the optical nerve head of each eye (laser parameters: $150 \mathrm{~mW} ; 100 \mathrm{msec}$; laser spot size, $0.05 \mathrm{~mm}$ ) using a diode laser system (Coherent, Inc., Santa Clara, CA, USA). Rupture of the Bruch's membrane was confirmed by the formation of a bubble immediately following laser application. The inclusion criteria of the lesions induced by laser, included the successful rupture of the Bruch's membrane and the absence of subretinal hemorrhage. The inclusion criteria of the present study included the formation of a bubble immediately following laser application and the absence of subretinal hemorrhage. Following full recovery from anesthesia, the mice were returned to the animal care facility.

Preparation of CRRR and treatment. The water extract $(0.6 \mathrm{~g} / 100 \mathrm{ml})$ of CRRR was prepared and packed by the Department of Pharmaceutical Sciences of the First People's Hospital (Shanghai, China) using a standardized procedure. Briefly, the lyophilized powder (Pingcheng Biotech Co. Ltd., Xi'an, China) was dissolved in distilled water $(0.6 \mathrm{~g} / 100 \mathrm{ml})$ prior to use. The mice were randomly divided into two groups (15 mice per group) and treated as follows: i) Control group, receiving laser treatment on day 0 and administered $0.9 \%$ saline $(1 \mathrm{ml} / 0.1 \mathrm{~kg}$, twice a day by oral gavage) between days 0 and 21; and (ii) CNV with CRRR group, receiving laser treatment on day 0 and administered CRRR $(1 \mathrm{ml} / 0.1 \mathrm{~kg}$, twice a day by oral gavage) between days 0 and 21 . The concentration and dosage of CRRR used in the present study were calculated based on the concentration used in the previously described pharmaceutical composition (patent no., WO2012079419).
Fundus fluorescein angiography (FFA). FFA of the CNV lesions was conducted using a Heidelberg Retina Angiograph 2 imaging system (Heidelberg Engineering, Inc., Vista, CA, USA). Fluorescein treatment (0.02 ml 25\% fluorescein; Alcon Laboratories, Irvine, CA, USA) was delivered by i.p. injection, on days 7, 14, and 21 following laser treatment. Timing began immediately after the fluorescein injection. Early-, middleand late-phase fundus angiograms were obtained at $45 \mathrm{sec}$, $2 \mathrm{~min}$ and $7 \mathrm{~min}$ after the fluorescein injection, respectively. The fluorescein leakage of each CNV lesion was graded by two independent retina specialists, unaware of the experimental design, according to a method previously described by Marneros et al (6). Briefly, the early-, middle- and late-phase angiograms were compared in order to determine the severity of the lesions: Grade 0 lesions showed no hyperfluorescence; grade 1 lesions exhibited hyperfluorescence without leakage; grade 2 a lesions exhibited hyperfluorescence in the early-phase or midtransit images and late-phase leakage; and grade $2 \mathrm{~b}$ lesions exhibited bright hyperfluorescence in the transit images and late-phase leakage beyond the treated areas. Only lesions with grade 2 b leakage were considered as clinically significant (Fig. 1).

Immunofluorescence and volumetric analysis. At 22 days after laser treatment, the mice were sacrificed by cervical dislocation and their eyes were enucleated. The eyes were fixed for $1 \mathrm{~h}$ in $4 \%$ paraformaldehyde. Then the anterior segments and the retinas were removed. The eye cups were washed with phosphate-buffered saline (PBS), then immersed in 2\% normal donkey serum (Sigma-Aldrich) and 1\% Triton X-100 (Invitrogen Life Technologies, Carlsbad, CA, USA) in PBS for 3-10 min. After incubation with isolectin B4 from Griffonia simplicifolia (AlexaFluor ${ }^{\circledR} 568 ; 25 \mu \mathrm{g} / \mathrm{ml}$; Invitrogen Life Technologies), phalloidin (AlexaFluor ${ }^{\circledR} 488 ; 5 \mathrm{U} / \mathrm{ml}$; Invitrogen Life Technologies) and Hoechst $33258(20 \mu \mathrm{g} / \mathrm{ml}$; Invitrogen Life Technologies), the eye cups were washed with PBS and cut with four peripheral radial cuts. The flat mounts were covered with a coverslip using ProLong ${ }^{\circledR}$ Gold antifade reagent (Invitrogen Life Technologies). A Zeiss LSM-510 Meta Laser Confocal microscope (Carl Zeiss, Inc., Thornwood, NY, USA) was used for sample observation and image capture. An image stack was collected for each lesion, which included the area from the retinal pigment epithelium (RPE) surface to the bottom of the lesion. Volumetric analysis of the images was performed using the Image $\mathbf{J}$ software (National Institutes of Health, Bethesda, MA, USA).

Quantitative polymerase chain reaction ( $q P C R)$. Total RNA was extracted from the posterior part of the eyeball (retina, RPE and choroid) at day 7 after the laser treatment using Isogen (Nippon Gene Co, Ltd., Tokyo, Japan) (7). Next, the total RNA was reverse-transcribed into cDNA using a Superscript III First Strand Synthesis system (Invitrogen Life Technologies) and random hexamer primers, according to the manufacturer's instructions. The gene expression levels were analyzed by qPCR using TaqMan ${ }^{\circledR}$ Gene Expression Assays (Applied Biosystems Life Technologies, Foster City, CA, USA). A realtime PCR cycler (ABI Prism 7800 Sequence Detection System; Applied Biosystems Life Technologies) was used for the experiment, according to the manufacturer's instructions. 

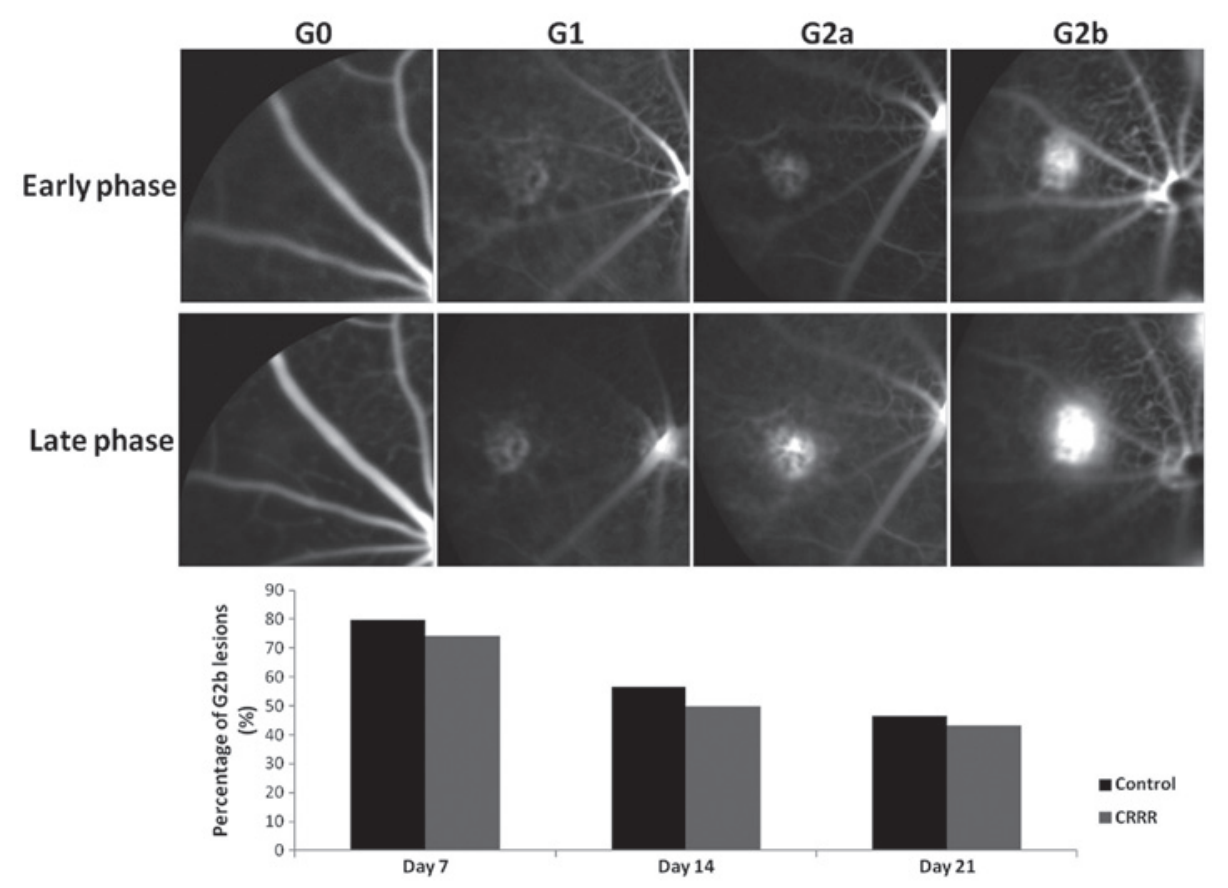

Figure 1. Grading system of lesions and the results of lesion grading. Grade $2 b$ (G2b) lesions were defined as lesions with clinically significant fluorescein leakage. The percentage of G2b lesion leakage was not found to be significantly different between the control and charred Radix et Rhizoma Rhei (CRRR)-treated groups at days 7, 14 and 21 after laser treatment (day 7, $\mathrm{P}=0.73$; day 14, $\mathrm{P}=0.69$; day 21, $\mathrm{P}=0.79$ ).

The assay identification numbers used in this study were as follows: interleukin-10 (IL-10), Mm00439614_m1; and VEGF, Mm00437304_m1. $\beta$-actin was used as the housekeeping gene, to normalize for differences in the efficiency of sample extraction or cDNA synthesis by reverse transcriptase. For relative quantification, the $2^{-\Delta \Delta \mathrm{Ct}}$ method was used.

Western blot analysis. Extracts from the retina-RPE-choroid complexes of five mice in each group were prepared for western blot analysis at day seven following laser photocoagulation, according to the method of Andrews and Faller (8). Briefly, total protein was obtained by lysing in a buffer containing $1 \mathrm{M}$ Tris- $\mathrm{HCl}$ (pH 7.5), 1\% Triton X-100, $1 \%$ nonidet $\mathrm{p}-40$, $10 \%$ sodium dodecyl sulfate, $0.5 \%$ sodium deoxycholate, $0.5 \mathrm{M}$ EDTA, $10 \mu \mathrm{g} / \mathrm{ml}$ leupeptin, $10 \mu \mathrm{g} / \mathrm{ml}$ aprotinin and $1 \mathrm{mM}$ phenylmethylsulfonyl fluoride. Protein was separated by $10 \%$ SDS-PAGE and transferred to polyvinylidine difluoride filter membranes (EMD Millipore, Bedford, MA, USA). The membranes were blocked in Tris-buffered saline (TBS) containing 5\% milk and $0.1 \%$ Tween-20 for $1 \mathrm{~h}$ at room temperature, then incubated at $4{ }^{\circ} \mathrm{C}$ overnight with the following primary antibodies: Rat monoclonal anti-IL-10 (1:200; sc-73309; Santa Cruz Biotechnology Inc., Dallas, TX, USA), rat polyclonal anti-VEGF (1:300; sc-507; Santa Cruz Biotechnology Inc.) and rat polyclonal anti- $\beta$-actin antibody (1:500; SAB2100037; Sigma-Aldrich). The membranes were then washed three times for $10 \mathrm{~min}$ each in TBS containing $0.1 \%$ Tween-20. Subsequently, the membranes were incubated with horseradish peroxidase-conjugated goat anti-rat immunoglobulin G secondary antibody (1:200; sc-45100; Santa Cruz Biotechnology, Inc.) for $2 \mathrm{~h}$. After three 10-min washes in TBS containing $0.1 \%$ Tween-20, the protein bands were visualized using an enhanced chemiluminescence system (Pierce
Biotechnologies Inc., Rockford, IL, USA). Densitometric analysis was performed using the Quantity One analysis software version 4.2.1 (Bio-Rad Laboratories, Hercules, CA, USA).

Statistical analysis. The data are expressed as the mean \pm standard deviation. Statistical analyses were performed using SPSS software version 19.0 (IBM, Armonk, NY, USA). Statistical significance was analyzed by one-way analysis of variance, followed by Student's t-test for CNV volume analysis, Mann-Whitney test for qPCR experiments and $\chi^{2}$ test for CNV leakage grading analysis. $\mathrm{P}<0.05$ was considered to indicate a statistically significant difference.

\section{Results}

Fluorescein leakage in laser treated eyes. The percentage of lesions showing a clinically significant leakage was determined by FFA (Fig. 1). In the control group, the percentage of lesions with grade $2 b$ fluorescein leakage was $79.65,56.67$ and $46.67 \%$ at days 7, 14 and 21 after laser treatment, respectively. In the CRRR-treated group, the percentage of lesions with grade $2 \mathrm{~b}$ leakage was $74.23,50.00$ and $43.33 \%$ at the same time points. No statistically significant differences were observed in the leakage grade between the control and CRRR-treated groups at days 7, 14 or 21 days after laser treatment (day 7, P=0.73; day $14, \mathrm{P}=0.69$; day $21, \mathrm{P}=0.79$ ).

CNV volume in flat mount. The CNV volumes were calculated from the sum of isolectin $\mathrm{B}_{4}$-labeled signals and were found to be $23457.45 \pm 227.42 \mu \mathrm{m}^{3}$ in the control group and $23245.43 \pm 290.12 \mu \mathrm{m}^{3}$ in the CRRR-treated group $(\mathrm{P}=0.14)$. No statistically significant difference was observed between the two groups ( $P>0.05$; Fig. 2). 

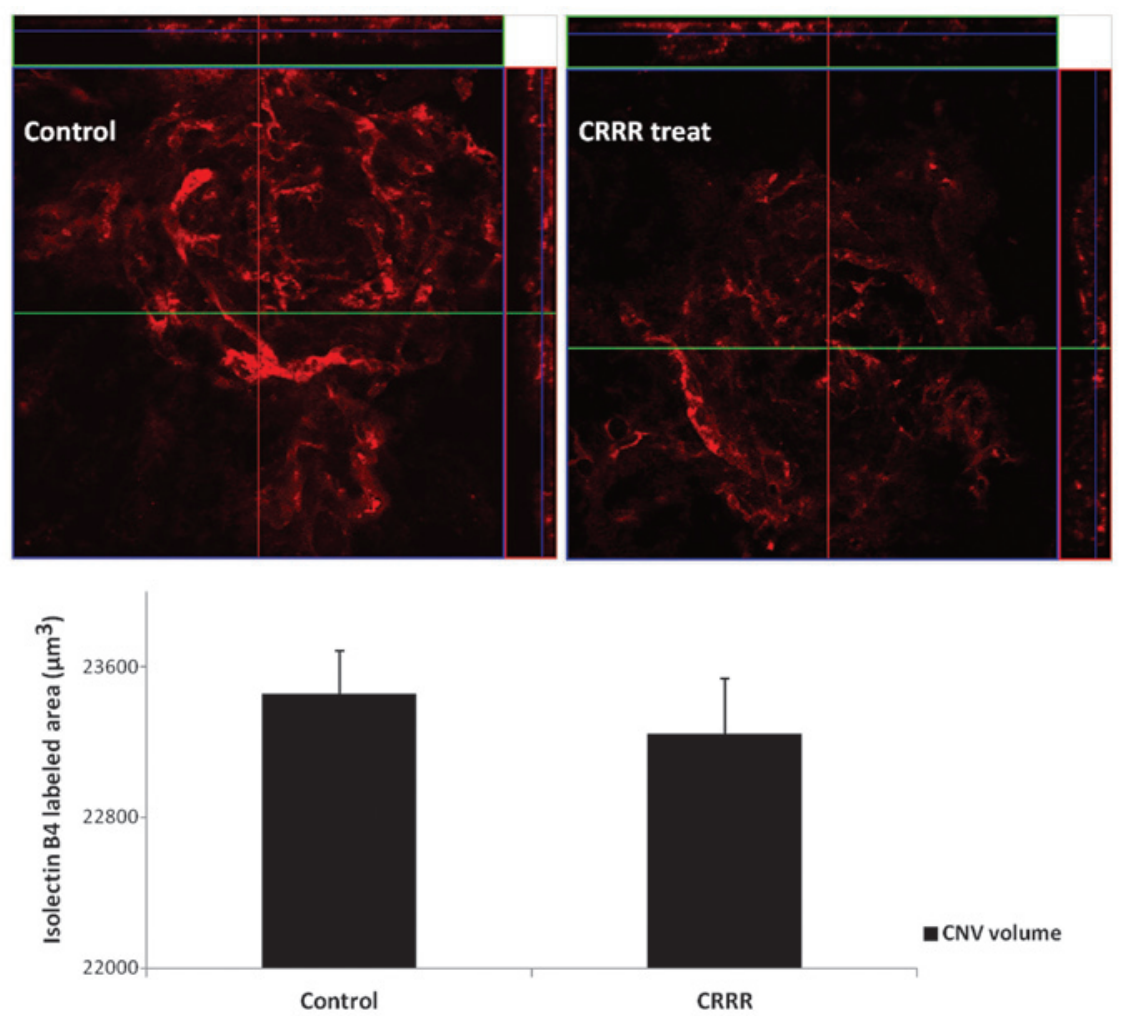

Figure 2. Z-stack images obtained from confocal microscopy analysis. Choroidal neovascularization (CNV) was labeled using isolectin $\mathrm{B}_{4}$ (red color; magnification, $\mathrm{x} 40$ ). The $\mathrm{CNV}$ volumes were calculated from the sum of isolectin $\mathrm{B}_{4}$-labeled signals and were found to be $23457.45 \pm 227.42 \mu \mathrm{m}^{3} \mathrm{in}$ the control group and $23245.43 \pm 290.12 \mu \mathrm{m}^{3}$ in the charred Radix et Rhizoma Rhei (CRRR)-treated group ( $\left.\mathrm{P}=0.14\right)$. Error bars indicated the mean \pm standard deviation. No statistically significant differences were observed between the two groups.

A

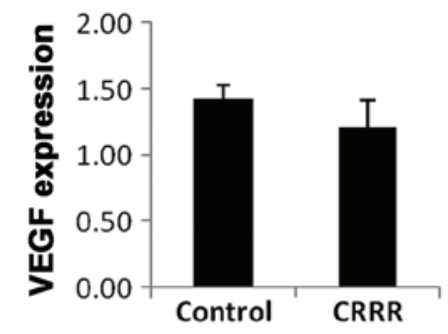

B
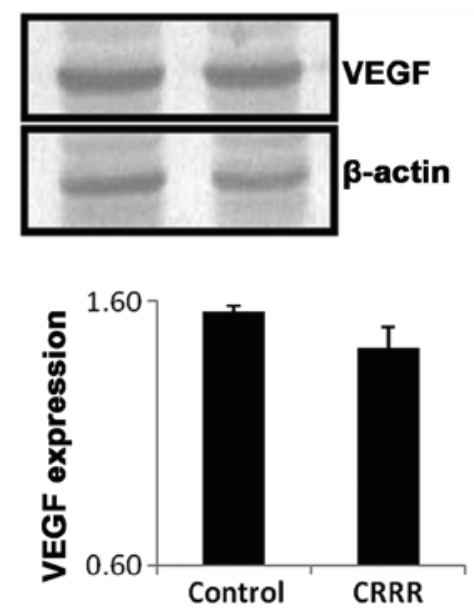
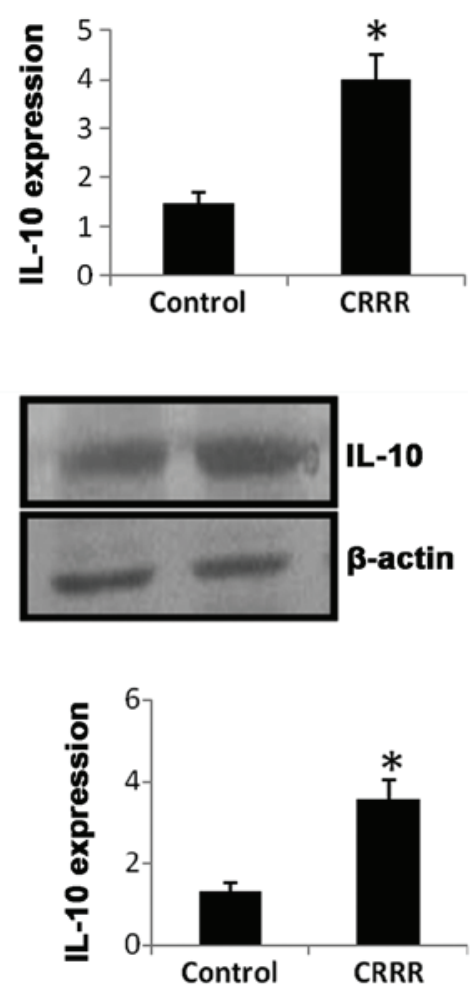

Figure 3. (A) Gene expression levels of vascular endothelial growth factor (VEGF) and interleukin-10 (IL-10), detected by quantitative polymerase chain reaction. At day 7 after laser treatment, the IL-10 mRNA expression level was significantly upregulated in the charred Radix et Rhizoma Rhei (CRRR)-treated group compared with the control group. No statistically significant difference was observed in the VEGF mRNA expression levels between the control and CRRR-treated groups (P>0.05). (B) Protein expression levels of VEGF and IL-10, determined by western blot analysis. The protein expression level of VEGF were not significantly different between the control and CRRR-treated groups (P>0.05), while the protein expression level of IL-10 was significantly upregulated in the CRRR-treated group compared with the control group. ${ }^{*} \mathrm{P}<0.05$, vs. control group. 
$q P C R$. At day 7 after laser treatment, the mRNA expression level of IL-10 was significantly upregulated in the CRRR-treated group, when compared with the control group $(\mathrm{P}=0.02)$. By contrast, no statistically significant difference was observed in the mRNA expression level of VEGF between the control and CRRR groups $(\mathrm{P}=0.65$; Fig. $3 \mathrm{~A})$.

Western blot analysis. At day 7 after laser treatment, the protein expression level of IL-10 was significantly increased in the CRRR-treated group, when compared with the control group $(\mathrm{P}=0.03)$. However, no statistically significant difference was observed in the VEGF protein expression level between the control and CRRR groups $(\mathrm{P}=0.74$; Fig. $3 \mathrm{~B})$.

\section{Discussion}

Wet AMD accounts for $90 \%$ of severe vision loss in AMD patients (9). The wet AMD subtype is characterized by the presence of exudate, bleeding, edema, pigment changes and $\mathrm{CNV}$ in the macular fovea. The development of CNV involves the appearance of hypoxia, inflammation, angiogenesis, fibrotic changes and endothelial cell-matrix interactions (10). The formation of CNV is influenced by a variety of factors, among which the expression of VEGF is critical; thus, VEGF-A neutralization has become the standard treatment method for CNV (11). Anti-inflammatory agents have also been used to treat CNV. However, corticosteroids are associated with severe adverse effects, such as elevated intraocular pressure, cataract development and endophthalmitis $(12,13)$. Although anti-VEGF agents can markedly improve the clinical outcome of wet AMD, they are unable to induce complete regression of the CNV membranes (14-16). Furthermore, anti-VEGF agents require repeated injections into the eye at intervals of four to six weeks in order to achieve an optimal outcome, which may increase the risk of ocular inflammation, retinal injury and endophthalmitis (14-16). Since VEGF plays an important role in retinal development and neuroprotection, anti-VEGF therapy may induce retinal damage following a long period of administration. Due to the systemic physiological role of VEGF, previous studies have suggested that the beneficial effects of VEGF antagonism in the eyes may result in adverse systemic reactions (17-19). Therefore, the safety of anti-VEGF treatment requires long-term observation. A previous study reported that only $30-40 \%$ of individuals experienced vision improvement following anti-VEGF treatment (20). Thus, VEGF inhibition or anti-inflammatory treatment alone may not be sufficient. Combination therapies have been explored in order to reduce the frequency of intravitreal anti-VEGF injections and the risk of relevant side-effects due to complete VEGF inactivity and steroid administration $(21,22)$. Investigations regarding other therapeutic strategies may prolong the interval of treatment and provide alternatives to the current CNV treatment $(5,23)$.

A novel TCM composition devised by Jin et al (4) (patent no. WO2012079419) is a prospective alternative therapy for wet AMD. The pharmaceutical composition consists of a number of TCM components, including Astragalus membranaceus Bunge, Angelica sinensis, Poria cocos Wolf, Fritillaria thunbergii, Panax pseudoginseng, CRRR, pollen Typhae and Curcuma aromatica Salisb. The compounds in the composition were shown to be capable of targeting the hallmarks of
AMD, including oxidative stress, RPE cytotoxicity, inflammation and vascular edema (5). The composition contains raw materials from herbs that can eliminate inflammation (25-30), modulate immunity (31-37), promote blood circulation in order to remove blood stasis $(38,39)$, prevent bleeding $(40,41)$ and promote the absorption of macular exudates, edema and hemorrhage, as well as reduce the leakage and area of CNV (42). Treatment with the composition was found to enhance and stabilize the vision of patients with AMD, while the CNV leakage was more significantly reduced compared with Avastin ${ }^{\circledR}$ treatment (anti-VEGF agent) (5). Initially, the composition did not exhibit a favorable clinical outcome, until Citrus reticulata (included in the original composition) was replaced with CRRR. Therefore, the present study applied the same concentration and dose of CRRR, as used in the clinical trial in order to treat laser-induced $\mathrm{CNV}$ in a murine model. The aim of the present study was to determine the role of CRRR in the composition. Radix et Rhizoma Rhei (RRR) is the Latin name for Da Huang, a Chinese herb, which is commonly known as rhubarb (Rheum palmatum L.). The root and underground stem of rhubarb, known as the rhizome, are often used to produce medicine, which can be used in a raw, alcohol-processed or carbonized form (43). RRR manifests a therapeutic function in the spleen, stomach, large intestine, liver and heart meridians. The herb mainly comprises derivatives of anthraquinone in a conjugated form of anthraquinone glycoside or diglycoside, as well as tannins and their analogues (43). RRR can purge intestinal stagnation, resolve inflammation, eliminate toxic substances, remove blood stasis and promote blood circulation (43). This herb has been previously used to treat conditions, such as constipation, acute intestinal obstruction, dysentery, bleeding symptoms, eye redness, throat soreness, abdominal abscess, jaundice, carbuncles and furuncles, irregular menstruation, traumatic injuries, rosacea, hyperlipidemia, renal failure, uremia, burns and scalds, and cholelithiasis $(43,44)$. Processed RRR has a reduced purgative effect and is typically used for the treatment of red eyes, sore throat, swollen gums, carbuncles and furuncles. Charred RRR (CRRR) is used for the treatment of bleeding symptoms and the prevention of bleeding, as well as the activation of local circulation (37,43-45). Therefore, CRRR can improve blood stasis, relieve swelling and pain, resolve inflammation and promote wound healing.

The present study focused on the effect of CRRR on CNV leakage and volume reduction. In order to uncover the underlying mechanisms of the CRRR effect, alterations in the expression levels of VEGF and IL-10 were determined. IL-10 is an anti-inflammatory and immunomodulatory cytokine produced by numerous human cell types, including monocytes, macrophages, B-lymphocytes and T-helper 2 cells (46). However, the role of IL-10 in CNV formation remains controversial. Apte et al (47) demonstrated that $\mathrm{CNV}$ was reduced in IL-10 knockout mice, when compared with wild type mice; thus, IL-10 may inhibit the recruitment of macrophages to the CNV area and promote CNV formation. By contrast, Hasegawa et al (48) indicated that IL-10 had anti-angiogenic properties in the formation of CNV (48). These results suggest that the effect of IL-10 on CNV may vary according to the expression level of IL-10 and the inflammatory microenvironment. In the present study, the intraocular expression level 
of IL-10 was found to be increased following treatment with CRRR. However, no other statistically significant differences were observed between the CRRR-treated and control group. Therefore, CRRR may exert anti-inflammatory and immunomodulatory effects in AMD patients. The effect of CRRR on bleeding symptoms may also play a role in the treatment of wet AMD. However, since lesions with hemorrhage were excluded from the current study, the effect of CRRR on CNV in the laser-induced CNV model may not mimic wet AMD efficiently. In the previous clinical trials, CRRR was used to treat patients with wet AMD synergistically with seven other components in the composition (4). Therefore, application of CRRR alone may not be sufficient to result in the regression of CNV. The differences between laser-induced CNV in mice and human CNV must also be considered. Furthermore, the experiments of the present study were performed in a murine model of acute inflammation; therefore, a chronic inflammatory model should be established in future studies.

TCM typically uses multiple herb combinations to treat and relieve the symptoms of various diseases. TCM therapies are attractive to patients since TCM tends to result in fewer and less severe side-effects compared with pure single drugs. Therefore, the multicomponent and synergistic nature of TCM should be considered in pharmaceutical development. Traditional herbs have been a major source of modern single-drug developments (49). The extractions used in target herbal medicine may result in the identification of promising novel active natural products, whereas synthetic analogues may be produced through verified pharmacological testing and bioactivity-directed fractionation and isolation of active ingredients (50). The active ingredients of a target drug may then be used in the subsequent drug development stage. The TCM composition, WO2012079419, consists of eight herbs that target different pathways and fit the mechanisms of AMD development. The pharmacological action of this composition may be due to a particular chemical or the complex interactions between the composition constituents. Previous studies have demonstrated that the herbal composition was able to simultaneously address numerous mechanisms of wet AMD (5). Modern analytical and pharmacological methods have been used to determine the properties of the herbal combinations in comparison with a single component. In addition, qualitative and quantitative analyses were performed to evaluate and guarantee the safety and efficacy of the composition (49). Modernization is important for the standardization of TCM and the establishment of qualitative and quantitative data on its active ingredients and bioactivity. In addition, the pharmaceutical composition may be prepared into various pharmaceutically acceptable forms, including decoction, tablet, capsule, bolus, oral liquid preparation and injection (50). Therefore, a non-invasive, efficient and safe therapy for AMD using the discussed TCM composition appears to be promising. In conclusion, CRRR did not appear to significantly inhibit CNV in this murine model. The function of CRRR in the pharmaceutical composition may be due to the anti-inflammatory and immunomodulatory effects of IL-10, as well as a synergistic effect with other components of the composition. A perspective, randomized, double-blind clinical trial should be performed in future studies to investigate the potential application of this composition in AMD.

\section{References}

1. Klein R, Peto T, Bird A and Vannewkirk MR: The epidemiology of age-related macular degeneration. Am J Opthalmol 137: 486-495, 2004.

2. Jian L, Panpan Y and Wen X: Current choroidal neovascularization treatment. Ophthalmologica 230: 55-61, 2013.

3. Campagne MVL, LeCouter J, Yaspan BL and Ye W: Mechanisms of age-related macular degeneration and therapeutic opportunities. J Pathol; 232: 151-164, 2014.

4. Jin M: Pharmaceutical composition for treating macular degeneration. International patent WO/2012/079419. Filed October 13, 2011; issued June 21, 2012.

5. Wang S and Cunnusamy K: Pharmaceutical composition for treating macular degeneration (W02012079419) Expert Opin Ther Pat 23: 269-272, 2013.

6. Marneros AG, She H, Zambarakji H, Hashizume H, Connolly EJ, Kim I, Gragoudas ES, Miller JW and Olsen BR: Endogenous endostatin inhibits choroidal neovascularization. FASEB J 21: 3809-3818, 2007.

7. Matsumara N, Kamei M, Tsujikawa M et al: Low-dose lipopolysaccharide pretreatment suppresses choroidal neovascularization via IL-10 induction. PLoS One7: e39890, 2012.

8. Andrews NC and Faller DV: A rapid micropreparation technique for extraction of DNA-binding proteins from limiting numbers of mammalian cells. Nucleic Acids Res 19: 2499, 1991.

9. Ferris FL III, Fine SL and Hyman L: Age-related macular degeneration and blindness due to neovascular maculopathy. Arch Ophthalmol 102: 1640-1642, 1984.

10. Veritti D, Sarao V and Lanzetta P: Neovascular age-related macular degeneration. Ophthalmologica 227: 11-20, 2012.

11. Li Y, Huang D, Xia X, Wang Z, Luo L and Wen R: CCR3 and choroidal neovascularization. PLoS One 6: e17106, 2011.

12. Arias 1, Garcia-Arumi J, Ramon JM, et al: Photodynamic therapy with intravitreal triamcinolone in predominantly classic choroidal neovascularization: one-year results of a randomized study. Opthalmology 113: 2243-2250, 2006.

13. Challa JK, Gillies MC, Penfold PL, et al: Exudative macular degeneration and intravitreal tramcinolone: 18 month follow up. Aust N Z J Opthalmol 26: 277-281, 1998.

14. Regillo CD, Brown DM, Abraham P, Yue H, Ianchulev T, Schneider S and Shams N: Randomized, double-masked, sham-controlled trial of ranibizumab for neovascular age-related macular degeneration: PIER Study year 1. Am J Ophthalmol 145: 239-248, 2008.

15. Nishijima K, Ng YS, Zhong L, Bradley J, Schubert W, Jo N, Akita J, Samuelsson SJ, Robinson GS, Adamis AP and Shima DT: Vascular endothelial growth factor-A is a survival factor for retinal neurons and a critical neuroprotectant during the adaptive response to ischemic injury. Am J Pathol 171: 53-67, 2007.

16. Robinson GS, Ju M, Shih SC, Xu X, McMahon G, Caldwell RB and Smith LE: Nonvascular role for VEGF: VEGFR-1, 2 activity is critical for neural retinal development. FASEB J 15: 1215-1217, 2001.

17. Ueta T, Yanagi Y, Tamaki Y and Yamaguchi T: Cerebrovascular accidents in ranibizumab. Ophthalmology 116: 362, 2009.

18. Campbell RJ, Bell CM, Paterson JM, Bronskill SE, Moineddin R, Whitehead M and Gill SS: Stroke rates after introduction of vascular endothelial growth factor inhibitors for macular degeneration: a time series analysis. Ophthalmology 119: 1604-1608, 2012.

19. Horsley MB, Mandava N, Maycotte MA and Kahook MY: Retinal nerve fiber layer thickness in patients receiving chronic anti-vascular endothelial growth factor therapy. Am J Ophthalmol; 150: 558-561, 2010.

20. Zhou QB, Anderson C, Zhang H, Li X, Inglis F, Jayagopal A and Wang SS: Repression of choroidal neovascularization through actin cytoskeleton pathways by mircoRNA-24. Mol Ther 22: 378-389, 2014.

21. Schaal S, Kaplan HJ and Tezel TH: Is there tachyphylaxis to intravitreal anti-vascular endothelial growth factor pharmacotherapy in age related macular degeneration? Ophthalmology 115: 2199-2205, 2008.

22. Dhalla MS, Shah GK, Blinder KJ, et al: Combined photodynamic therapy with verteporfin and intravitreal bevacizumab for choroidal neovascularization in age-related macular degeneration. Retina 26: 988-993, 2006.

23. Ebrahim S, Peyman GA and Lee PJ: Applications of liposomes in ophthalmology. Surv Ophthalmol 50: 167-182, 2005. 
24. Ko JK and Chik CW: The protective action of radix Astragalus membranaceus against hapten-induced colitis through modulation of cytokines. Cytokine 47: 85-90, 2009.

25. Han $\mathrm{C}$ and Guo J: Antibacterial and anti-inflammatory activity of traditional Chinese herb pairs, Angelica sinensis and Sophora flavescens. Inflammation 35: 913-919, 2012.

26. Chang SH, Choi Y, Park JA, et al: Anti-inflammatory effects of BT-201, an n-butanol extract of Panax notoginseng, observed in vitro and in a collagen-induced arthritis model. Clin Nutr 26 : 785-791, 2007.

27. Fuchs SM, Heinemann C, Schliemann-Willers S, et al: Assessment of anti-inflammatory activity of Poria cocos in sodium lauryl sulphate-induced irritant contact dermatitis. Skin Res Technol 12: 223-227, 2006.

28. Cho IH, Lee MJ, Kim JH, Han NY, Shin KW, Sohn Y and Jung HS: Fritillaria ussuriensis extract inhibits the production of inflammatory cytokine and MAPKs in mast cells. Biosci Biotechnol Biochem 75: 1440-1445, 2011.

29. Mandal MN, Patlolla JM, Zheng L, et al: Curcumin protects retinal cells from light-and oxidant stress-induced cell death. Free Radic Biol Med 46: 672-679, 2009.

30. Lee HS, Lee MJ, Kim H, et al: Curcumin inhibits TNFalpha-induced lectin-like oxidised LDL receptor-1 (LOX-1) expression and suppresses the inflammatory response in human umbilical vein endothelial cells (HUVECs) by an antioxidant mechanism. J Enzyme Inhib Med Chem 25: 720-729, 2010.

31. Yang WJ, Li DP, Li JK, et al: Synergistic antioxidant activities of eight traditional Chinese herb pairs. Biol Pharm Bull 32: 1021-1026, 2009

32. Yang X, Zhao Y, Zhou Y, et al: Component and antioxidant properties of polysaccharide fractions isolated from Angelica sinensis (OLIV.) DIELS. Biological Pharmaceutical Bulletin 30 1884-1890, 2007

33. Jang YJ, Kim ME and Ko SY: n-Butanol extracts of Panax notoginseng suppress LPS-induced MMP-2 expression in periodontal ligament fibroblasts and inhibit osteoclastogenesis by suppressing MAPK in LPS-activated RAW264.7 cells. Arch Oral Biol 56: 1319-1327, 2011.

34. Choi RC, Jiang Z, Xie HQ, et al: Anti-oxidative effects of the biennial flower of Panax notoginseng against $\mathrm{H}_{2} \mathrm{O}_{2}$-induced cytotoxicity in cultured PC12 cells. Chin Med 5: 38, 2010.

35. Kanayama H, Adachi N and Togami M: A new antitumor polysaccharide from the mycelia of Poria cocos Wolf. Chem Pharm Bull 31: 1115-1118, 1983.

36. Seo JS, Jung EY, Kim JH, et al: A modified preparation (LMK03) of the oriental medicine Jangwonhwan reduces Abeta (1-42) level in the brain of Tg-APPswe/PS1dE9 mouse model of Alzheimer disease. J Ethnopharmacol 130: 578-585, 2010.
37. Li Z, Xia X, Zhang S, et al: Up-regulation of Toll-like receptor 4 was suppressed by emodin and baicalin in the setting of acute pancreatitis. Biomed Pharmacother 63: 120-128, 2009.

38. Zhang L, Yang Y, Wang Y and Gao X: Astragalus membranaceus extract promotes neovascularisation by VEGF pathway in rat model of ischemic injury. Pharmazie 66: 144-150, 2011

39. Lam HW, Lin HC, Lao SC, et al: The angiogenic effects of Angelica sinensis extract on HUVEC in vitro and zebrafish in vivo. J Cell Biochem 103: 195-211, 2008.

40. Yeh JC, Cindrova-Davies T, Belleri M, et al: The natural compound n-butylidenephthalide derived from the volatile oil of Radix Angelica sinensis inhibits angiogenesis in vitro and in vivo. Angiogenesis 14: 187-197, 2011.

41. Ohkura N, Tauchi C, Nakayama A and Atsumi G: Pollen Typhae is a rapid hemostyptic. Blood Coagul Fibrinolysis 23: 254-255, 2012.

42. Ding JD, Johnson LV, Herrmann R, et al: Anti-amyloid therapy protects against retinal pigmented epithelium damage and vision loss in a model of age-related macular degeneration. Proc Natl Acad Sci USA 108: E279-E287, 2011.

43. Lei ZQ: Chinese herbs/Da Huang, In: Chinese Medicine. Chen SY and Gao XY (eds). 1st edition. Shanghai Science and Technology Publisher, Shanghai, pp176-182, 1998 (In Chinese).

44. Gan T, Liu YD, Wang Y and Yang J: Traditional Chinese Medicine herbs for stopping bleeding from haemorrhoids. Cochrane Database Syst Rev 10: CD006791, 2010.

45. Kumar A, Dhawan S and Aggarwal BB: Emodin (3-methyl-1,6,8-trihydroxyanthraquinone) inhibits TNF-induced $\mathrm{NF}-\kappa \mathrm{B}$ activation, I $\kappa \mathrm{B}$ degradation and expression of cell surface adhesion proteins in human vascular endothelial cells. Oncogene 17: 913-918, 1998.

46. Saraiva M and O'Garra A: The regulation of IL-10 production by immune cells. Nat Rev Immunol 10: 170-181, 2010.

47. Apte RS, Richter J, Herndon J and Ferguson TA: Macrophages inhibit neovascularization in a murine model of age-related macular degeneration. PLoS Med 3: e310 2006.

48. Hasegawa E, Oshima Y, Takeda A, Saeki K, Yoshida H, et al: IL-27 inhibits pathophysiological intraocular neovascularization due to laser burn. J Leukoc Biol 91: 267-273, 2012.

49. Tsang KW, Lam CL, Yan C, et al: Coriolus Versicolor Polysaccharide Peptide slows progression of advanced non-small cell lung cancer. Respir Med 97: 618-624, 1997.

50. Lee KH: Research and future trends in the pharmaceutical development of medicinal herbs from Chinese medicine. Public Health Nutr 3: 515-522, 2000. 